Article

\title{
A Dynamic Model for the Normal Impact of Fly Ash Particle with a Planar Surface
}

\author{
Ming Dong, Jian Han, Sufen Li * and Hang Pu
}

School of Energy and Power Engineering, Dalian University of Technology, Dalian 116024, China; E-Mails: dongming@dlut.edu.cn (M.D.); hanjian0822@163.com (J.H.); stingph42@gmail.com (H.P.)

* Author to whom correspondence should be addressed; E-Mail: lisuf@dlut.edu.cn;

Tel./Fax: +86-411-8470-8540.

Received: 16 May 2013; in revised form: 3 July 2013 / Accepted: 31 July 2013 /

Published: 20 August 2013

\begin{abstract}
The rebound behavior of fly ash particles normally impacting a planar surface is investigated by using a dynamic model. The three forms of soft sphere physical model are obtained using static/quasi-static contact mechanics and energy dissipation theory. The influences of the particle size, the incident velocity of the particle on the damping coefficient and the impact contact time are all examined. We also predict the critical velocity for three particle sizes. It is found that the variation of the damping coefficient $(\eta)$ with the normal incident velocity $\left(v_{i n}\right)$ can be roughly divided into the three parts. In the first part, $\eta$ decreases with increasing $v_{i n}$. In the second part, $\eta$ is little changed with increasing $v_{i n}$. In the third part, $\eta$ rapidly increases with increasing $v_{i n}$. For smaller impact velocities, the viscoelastic effect plays a dominant role in the impact process, while for higher incident velocities; the energy dissipation depends mainly on plastic deformation. In addition, the critical velocity shows a distinct dependence on the particle size. Finally, the contact displacement-contact time curves are examined. The work provides a solid basis for the development of a discrete-element-method approach to study ash deposition.
\end{abstract}

Keywords: ash deposition; energy dissipation; contact; impact; ash particle

\section{Introduction}

In industrial facilities using solid pulverized fuels, fouling and slagging are considered two of the most critical issues to be tackled. In general, any stream in which particles are advected may show a 
deposition behavior [1]. Sometimes, the deposit build-up may severely decrease the performance of the facility [2]. Moreover, the ash deposition has undesirable consequences on heat transfer, increases pressure drop, interferes aerodynamically with the flow and causes heat estranger pipe corrosion through the uptake and retention of corrosive, condensable vapors by the fouled surface. All these effects translate into overall plant efficiency losses, high operating/maintenance costs and reduced availability of the units [3]. Ash deposition is defined as the accumulation of ash particles on a heat exchange surface that is essentially a particle-surface process. Modeling of the process of particle-surface impact is complicated, primarily because it is dynamic and nonlinear [4].

To better understand these problems, an appropriate dynamic model describing the impact between a fine particle and a flat surface is essential. The dynamic impact procedure has been extensively studied through experiments. Dahneke studied the normal impact of polystyrene latex microspheres with surfaces under vacuum conditions [5,6]. However, the accurate measurement of final bouncing velocity near the critical velocity, where the adhesion effect plays a predominant role, was not achieved in that work. Rogers and Reed measured critical impact velocity for large $(15-40 \mu \mathrm{m})$ copper microspheres using a high speed camera. They evaluated an elastic-plastic impact model for particle adhesion [7]. Measurements of individual normal and oblique impacts of microspheres with planar surfaces are described and analyzed by Dunn et al. [8]. Their experimental results were similar to those of previous studies using monodisperse spheres. Kim and Dunn [9] provided direct imaging results of microparticle collisions. 40- $\mu \mathrm{m}$ diameter Ag-coated glass particles were dispersed and vertically dropped on a silica target plate. The collisions under standard atmospheric conditions were resolved with a digital high-speed technique black-light illumination. However, one of the most comprehensive experimental investigations of small particles impacting surfaces at normal incidence is that of Wall et al. [10]. They measured incoming and rebounding particle velocities to within several particle diameters of the impaction surface using a laser Doppler velocimetry system. At low velocity $(<20 \mathrm{~m} / \mathrm{s})$, the ratio of rebound to impact velocity was sensitive to target material, decreasing with impact velocity due to the adhesion surface energy, while for higher particle velocities, the ratio of rebound to impact velocity was insensitive to the target material.

From the theoretical aspect, on the other hand, analytical models have been investigated to understand the impact process. Relevant models can be generally categorized into kinetic models and dynamic models [11-13]. Kinetic models are based on two fundamental assumptions, namely, that the energy losses are due to the process of material deformation and the process of adhesion $[4,7,10,14-16]$. Kinetic models can predict the capture velocity, the relationship between restitution coefficient and incident velocity, and critical velocities. Kinetic models have been extensively used in studies such as hard-sphere discrete element method (DEM) simulations [12,17]. However, kinetic models can hardly reveal the dynamic features of the impact process, in terms of time-variant contact force, particle velocity, deformation and the relationships between them, etc. [13]. Dynamic models are described by the full equation of motion for all bodies affected by the impact. Such models provide information not only on the macroscopic parameters (including restitution coefficient and capture velocity) based on more in-depth contact mechanics [18], but also can extensively predict interparticle interactions in the DEM simulation of fine particulate flows [19-21].

Brach and Dunn proposed a dynamic model for adhesive impact based on the Hertzian contact theory under adhesion loading in earlier work [11]. Energy is dissipated through material deformation damping 
and adhesion damping. These energy losses are assumed to be independent. The model has been extensively used in subsequent work. For example, the model extended to more complicated situations by Cheng, Brach, and Dunn is presented in both dimensional and nondimensional forms [22]. The model has been developed for implementation with computational fluid dynamic software by Kim and Dunn, to investigate the variation of the normal coefficient of restitution against the incident velocity and material properties [23]. Thornton and Ning [18] considered the normal elastic-perfectly plastic impact considering the effect of interfacial adhesion. Based on the Johnson-Kendall-Roberts (JKR) theory of adhesion [24], they derived an analytical solution of restitution coefficient for the normal impact of adhesive, elastic-perfectly plastic spheres. Elhady, Rindt, Wijers and Steenhoven [25] developed a numerical model based on DEM and simulated the interactions between a particle hitting a bed of particles. The contact forces between the colliding particles are based on the Thornton and Ning model. The numerical model can predict the critical velocity at which an incident particle starts to stick, rebound or remove other particles from a bed of particles. Recently, Marshall [20], and Shuiqing and Marshall [21] proposed a dynamic model for soft-sphere DEM based on JKR theory, and successfully applied it to the three-dimensional simulations of micro-particle deposition on surfaces such as a wall or a cylinder in low-Reynolds number dispersed flows.

Despite the above developments on the study of the impact theoretical problems of particles with a substrate, there are still many important issues that remain unresolved. For example, practical ash particles impact a flat and powdery substrate. In addition, the effects of the temperatures of particle and substrate on the rebound characteristic of particles need to be investigated in detail. Therefore, the present work is the basis for a deeper study of the above listed problems, and it will have a guiding significance to the further development of this subject. An experimental set-up was developed to study the normal impact of particles with a planar surface and these are described in Section 2. Dynamic models and energy dissipation theory of the normal impact of particles with a planar surface are introduced (Section 3). In Section 4, firstly the variations of restitution coefficient versus incident velocity are discussed with experiments. Secondly, the variations of the damping coefficient versus normal incident velocity are developed based experimental results and numerical calculation. Thirdly the critical velocity is predicted with experiment and numerical calculation. Finally, the contact displacement-contact time curves are examined.

\section{Description of Experiments}

An experimental facility was developed to investigate the impact of fly ash particle with planar surfaces. A schematic of the experimental facility with the configuration used for normal impact measurements is shown in Figure 1. Nitrogen was drawn through a fluidized bed particle generator and carries the fly ash particles into the test section (the incident velocity of $0-16 \mathrm{~ms}^{-1}$ ). The test section includes a circular planar surface with a diameter of $2 \mathrm{~mm}$. The planar surface was made of stainless steel and was oriented parallel to the horizontal. The incident and rebound normal velocity components of fly ash particles were recorded using a digital high-speed camera (Phantom V12.1, Vision Research Inc., Wayne, NJ, USA) at a frame rate of 80,000 frames/s, the resolution of $256 \times 128$ and the exposure time of $11.93 \mu \mathrm{s}$. Optical lenses (Tokina AT-XM 100 PROD, Kenko Tokina Co. Ltd., Tokyo, Japan) were attached to the camera to achieve enough magnification and record individual particle motion. A fiber optic solar light source (XD-300 Xenon Lamp, XD-300 xenon lamp, Alltion Co. Ltd., Guangxi, 
China) was used to backlight the fly ash particle impact events. The camera output is connected to a digitizer and a frame grabber in a personal computer for image analysis. All experiments were conducted at a temperature of $\sim 22{ }^{\circ} \mathrm{C}$ and a relative humidity of $25 \% \pm 3 \%$. Experimental repeatability was assessed by repeating the experiment under the same conditions.

In the present study the samples of fly ash particles were taken from the dust in front of the boiler from a power plant and was used as impacting particles. Before carrying out an experiment, the particles were stored in a stove at $110^{\circ} \mathrm{C}$ to minimize problems with agglomeration.

The fly ash was sieved to separate the different size ranges. The crystal phases and weight percentages of the fly ash was investigated using XRD analysis. The fly ash was divided into the sample for XRD. Its scans were from $10^{\circ}$ to $70^{\circ}$ at a scanning speed of $1.25^{\circ} 2 \theta / \mathrm{min}$. Through XRD analysis, the crystallinity data of fly ash particles can be obtained. Then the data can be used to estimate the Young's modulus of fly ash particles [26].

Figure 1. Schematic of the experimental configuration with the high-speed camera system used in the normal impact experiments.

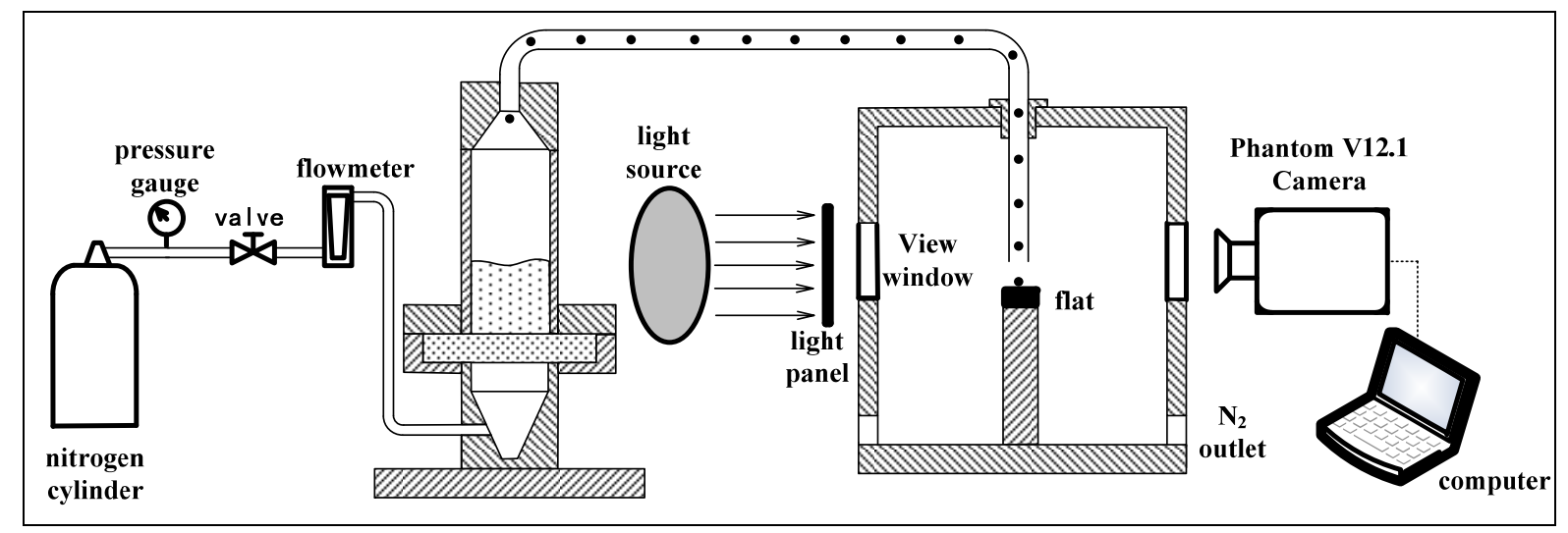

From the XRD analysis, it is found that the fly ash is mainly made of either quartz, $\mathrm{Q},\left(\mathrm{SiO}_{2}\right)$ or mullite, $\mathrm{M},\left(3 \mathrm{Al}_{2} \mathrm{O}_{3} \cdot 2 \mathrm{SiO}_{2}\right)$. The weight percentage of crystallinity was calculated based on the XRD result which was obtained by the software of MDI Jade 5.0. This paper assumes that the crystalline phase of fly ash only consist of quartz and mullite. Since the density of quartz, mullite ,and glassy phase are $2.65,2.80$ and $2.5 \mathrm{~g} \cdot \mathrm{cm}^{-3}$ [27], respectively, then the volume fractions of quartz, mullite, and glassy phase can be obtained. The volume fractions of all crystalline phases (quartz and mullite ) which are obtained from the results of quantitative XRD analysis. The volume fractions of all crystalline phases (quartz and mullite) which are obtained from the results of quantitative XRD analysis are shown in Table 1.

Table 1. Volume fractions of total crystalline phase, quartz, and mullite in fly ash of different sizes.

\begin{tabular}{cccc}
\hline Particle size (mesh) & Total cryscrystalline phase (vol\%) & Quart (zvol\%) & Mullite (vol\%) \\
\hline $104 \mu \mathrm{m}(230)$ & 67.31 & 10.19 & 57.12 \\
$96 \mu \mathrm{m}(270)$ & 55.02 & 6.29 & 48.73 \\
$88 \mu \mathrm{m}(325)$ & 49.28 & 1.65 & 47.63 \\
\hline
\end{tabular}

The Young's modulus of fly ash was estimated using the parallel model of rules of mixtures [26]. The parallel model is written as: 


$$
E_{f l y \text { ash }}=E_{g} V_{g}+E_{m} V_{m}+E_{q} V_{q}
$$

where $E$ is the Young's modulus; $V$ is volume fraction; and subscripts $g, m$, and $q$ denote glass, mullite, and quartz, respectively. The modulus of quartz and mullite were taken as $94 \mathrm{GPa}$ and $230 \mathrm{GPa}$, respectively, and the average moduli of glassy phase was taken as $73 \mathrm{GPa}$ [28]. According to this, the Young's moduli of $104 \mu \mathrm{m}, 96 \mu \mathrm{m}, 88 \mu \mathrm{m}$ fly ash particles are $164.81 \mathrm{GPa}, 150.82 \mathrm{GPa}$ and $148.13 \mathrm{GPa}$, respectively.

\section{Theoretical}

\subsection{Dynamic Model}

Dynamic models generally describe the impact process through the combination of the static contact theory and appropriate dissipation mechanisms. The mechanical description of static contact theory between the interacting particles is the basis of particle collision dynamics. The contact forces developed during collision of two particles are shown in Figure 2. The contact force depends on the impaction phase and the velocity of the impacting particles. Impaction between particles can be divided into two consecutive phases, the approach and the restitution phase. The approach phase ends when the two bodies have a relative normal velocity equal to zero as a result of impact. The motion equation of the particle is given by:

$$
m * \frac{d^{2} \delta}{d t^{2}}+\eta \frac{d \delta}{d t}+F_{t o t}=0
$$

where $\eta$ is the damping coefficient. The total force $F_{\text {tot }}$ is the sum of the forces exerted on the particle by other particles, defined as $F_{t o t}=F_{r}+F_{a}$, where $F_{r}$ and $F_{a}$ are the repulsive force and attractive force components of the total force, respectively. $m^{*}$ is the related to the particle masses $m_{i}$ by the equation $m^{*}=1 / m_{1}+1 / m_{2} . \delta$ is the interpenetration distance between the interacting particles as shown in Figure 2, and defined as $\delta=r_{1}+r_{2}-d$. $a$ is the contact radius.

Figure 2. Schematic of contact model between two particles.

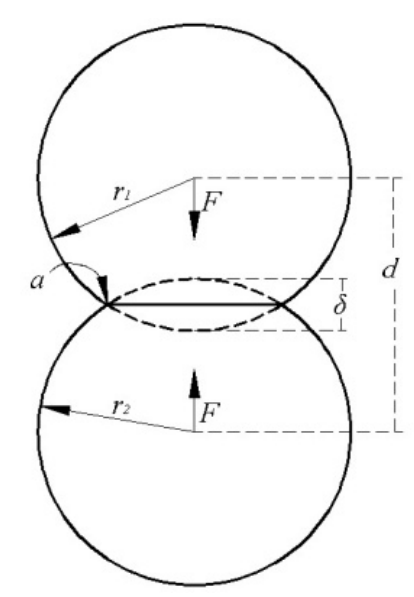

The normal contact force developed during the approach phase of a purely elastic collision is described by the Hertz theory [29]. The Hertzian pressure distribution over the contact area of radius $a$ is: 


$$
p=p_{0}\left(1-\frac{r^{2}}{a^{2}}\right)^{1 / 2}
$$

where $p_{0}$ is the maximum contact normal force; $r$ is the distance from the center of the contact area; $p$ is the contact normal force at the point $r ; a$ is the contact radius.

The maximum contact normal pressure $p_{0}$ and contact radius $a$ are given by:

$$
\begin{gathered}
p_{0}=\frac{2}{\pi} E^{*}\left(\frac{\delta}{R^{*}}\right)^{1 / 2} \\
a^{2}=R^{*} \delta
\end{gathered}
$$

In the above equations, $R^{*}$ and $E^{*}$ are defined as:

$$
\begin{gathered}
\frac{1}{R^{*}}=\frac{1}{r_{1}}+\frac{1}{r_{2}} \\
\frac{1}{E^{*}}=\frac{1-v_{1}^{2}}{E_{1}}+\frac{1-v_{2}^{2}}{E_{2}}
\end{gathered}
$$

with the subscripts 1 and 2 representing the interacting particle 1 and 2. $E$ is the Young's modulus of particle and $v$ is the Poisson's ratio.

Then Hertz total force $\mathrm{F}^{\mathrm{H}}$ is obtained by integration of the pressure over the contact region, which gives:

$$
\begin{aligned}
F_{t o t}{ }^{H} & =\int_{0}^{a} p(r) 2 \pi r d r=\int_{0}^{a} p_{0}\left(1-\frac{r^{2}}{a^{2}}\right)^{1 / 2} 2 \pi r d r \\
& =\int_{0}^{a} p_{0} \pi\left(1-\frac{r^{2}}{a^{2}}\right)^{1 / 2} d r^{2}=-\int_{0}^{a} p_{0} \pi\left(1-\frac{r^{2}}{a^{2}}\right)^{1 / 2} d\left(1-\frac{r^{2}}{a^{2}}\right) \\
& =-\left.\frac{2}{3} p_{0} \pi\left(1-\frac{r^{2}}{a^{2}}\right)^{3 / 2}\right|_{0} ^{a}=\frac{2}{3} p_{0} \pi a^{2}
\end{aligned}
$$

Substituting Equations (4) and (5) into Equation (8) we obtain:

$$
F_{\text {tot }}{ }^{H}=\frac{2}{3} \frac{2}{\pi} E^{*}\left(\frac{\delta}{R^{*}}\right)^{1 / 2} R^{*} \delta \pi=\frac{4}{3} E^{*} R^{* 1 / 2} \delta^{3 / 2}
$$

The Hertz model only considers the repulsive force between the interacting particles. The actual impact process presents the attraction force since the surface of the interacting particle has a surface energy. Bradley [30], DMT [31] and Maugis-Dugdale [32] (DMT model) developed the dynamics model of a normal impact, which considers repulsive force and attractive force of the total force between the interacting particles. The expression of the repulsive force $F_{r}{ }^{D M T}$ is derived from Hertz theory as follows:

$$
F_{r}^{D M T}=\frac{4}{3} E^{*} R^{* 1 / 2} \delta^{3 / 2}
$$

while the attractive force is idealized by an annular force at the perimeter of the contact area as:

$$
F_{a}^{D M T}=-2 \pi w R^{*}
$$


where, the adhesion work $w$ is defined by $w=\gamma_{1}+\gamma_{2}+\gamma_{12}, \gamma_{i}$ represents the surface energy of particle or the surface and $\gamma_{12}$ is interfacial energy. Practically $\gamma_{12}$ can be approximated by $w=\gamma_{1}+\gamma_{2}-2\left(\gamma_{1} \gamma_{2}\right)^{1 / 2}$ and hence leads to $w=\left(\gamma_{1} \gamma_{2}\right)^{1 / 2}$.

A similar strategy was also adopted by Brach and Dunn [11], the expressions of the repulsive force $F_{r}^{B D}$ and the attractive force $F_{a}^{B D}$ give:

$$
F_{r}^{B D}=\frac{4}{3} E^{*} R^{* 1 / 2} \delta^{3 / 2}
$$

while the attractive force is idealized by a line force at the perimeter of the contact area as:

$$
\begin{gathered}
F_{a}^{B D}=-2 \pi a f=-2 \pi f R^{*_{1 / 2}} \delta^{1 / 2} \\
f=\left(\frac{6 w^{2} R^{*} E^{*}}{\pi}\right)^{1 / 3}
\end{gathered}
$$

The expressions of $F_{r}$ and $F_{a}$ are derived from JKR theory as follows. Under a contact state with overlap $\delta$ and contact radius $a$, the relation between total force $F_{\text {tot }}{ }^{J K R}$ imposed on a particle and radius $a$ is given as:

$$
a^{3}=\frac{R}{K}\left(F+3 \pi w R^{*}+\sqrt{6 \pi w R^{*} F+\left(3 \pi w R^{*}\right)^{2}}\right)
$$

The relation between $F_{t o t}{ }^{J K R}$ and $\delta$ is implicit. However, $\delta$ varies with $a$ through:

$$
\delta=\left[a^{2}-\left(\frac{8 \pi w R^{* 2}}{3 K} a\right)^{1 / 2}\right] / R
$$

which enables establishing the $\mathrm{F} \sim \delta$ relationship implicitly. The stress distribution upon the particle within the contact area is given by [21,29]:

$$
p=p_{0}\left(1-r^{2} / a^{2}\right)^{1 / 2}-\frac{p_{0}^{\prime}}{\left(1-r^{2} / a^{2}\right)^{1 / 2}}
$$

where $p_{0}=3 a K / 2 \pi R^{*} ; p_{0}^{\prime}=\sqrt{3 w K / 2 \pi a}$. The stress is compressive within a circle of radius $r_{c}$ and tensile in the outer annulus $\left(r_{c}<\mathrm{r} \leq a\right)$. By setting Equation (17) to zero, we have $r_{c} / a=\sqrt{1-q_{0}}$, where $q_{0}=p_{0}^{\prime} / p_{0}$. Then $F_{r}^{J K R}$ is obtained by integration of the tensile stress over the annular region, which gives:

$$
F_{a}^{J K R}=-\frac{4}{3} q_{0}^{3 / 2} \pi a^{2} p_{0}
$$

Similary, $F_{\text {tot }}^{J K R}$ is obtained as:

$$
F_{\text {tot }}{ }^{J K R}=\left(\frac{2}{3}-2 q_{0}\right) \pi a^{2} p_{0}
$$

and hence:

$$
F_{r}^{J K R}=\left(\frac{2}{3}-2 q_{0}+\frac{4}{3} q_{0}^{3 / 2}\right) \pi a^{2} p_{0}
$$


Note that Equation (17) requires $0 \leq q_{0} \leq 1$ and hence $a \geq \sqrt{2 \pi w R^{* 2} / 3 K}$. Consequently, there will be tensile stress left for $a<\sqrt{2 \pi w R^{* 2} / 3 K}$ with $F_{r}=0$ and $F_{t o t}=F_{a}$.

The relationship between contact force and particle deformation can be utilized to understand the differences between Hertz, DMT, BD and JKR. In order to do this, we normalize force and overlap by:

$$
\bar{a}=a\left(\frac{K}{\pi w R^{* 2}}\right)^{1 / 3}, \bar{F}=\frac{F}{\pi w R^{*}}, \bar{\delta}=\left(\frac{16 E^{* 2}}{9 \pi^{2} w^{2} R^{*}}\right)^{1 / 3} \delta
$$

Then for Hertz, DMT, BD and JKR models, we have:

$$
\bar{F}_{\text {tot }}^{H}=\bar{F}_{r}^{H}=\bar{\delta}^{3 / 2}
$$

and:

$$
\bar{F}_{r}^{D M T}=\bar{\delta}^{3 / 2}, \bar{F}_{a}^{D M T}=-2, \bar{F}_{t o t}^{D M T}=\bar{F}_{r}^{D M T}+\bar{F}_{a}^{D M T}=\bar{\delta}^{3 / 2}-2
$$

and:

$$
\bar{F}_{r}^{B D}=\bar{\delta}^{3 / 2}, \bar{F}_{a}^{B D}=-6^{2 / 3} \bar{\delta}^{1 / 2}, \bar{F}_{t o t}^{B D}=\bar{F}_{r}^{B D}+\bar{F}_{a}^{B D}=\bar{\delta}^{3 / 2}-6^{2 / 3} \bar{\delta}^{1 / 2}
$$

For the JKR model, we have to separately consider the ranges of $\bar{a} \leq(2 / 3)^{1 / 3}$ and $\bar{a}>(2 / 3)^{1 / 3}$ such that:

$$
\bar{F}_{r}^{J K R}=\bar{a}^{3}-\sqrt{6} \bar{a}^{-3 / 2}+2(2 \bar{a} / 3)^{3 / 4}, \bar{F}_{a}^{J K R}=-2(2 \bar{a} / 3)^{3 / 4}, \bar{F}_{\text {tot }}^{J K R}=\bar{a}^{3}-\sqrt{6} \bar{a}^{3 / 2}
$$

for $\bar{a}>(2 / 3)^{1 / 3}$, and:

$$
\bar{F}_{r}^{J K R}=0, \bar{F}_{a}^{J K R}=\bar{F}_{t o t}^{J K R}=\bar{a}^{3}-\sqrt{6} a^{-3 / 2}
$$

for $6^{-1 / 3} \leq \bar{a} \leq(2 / 3)^{1 / 3}$. On the other hand we have:

$$
\bar{\delta}=\bar{a}^{2}-2(2 \bar{a} / 3)^{1 / 2}
$$

$\bar{F}_{r}, \bar{F}_{a}$ and $\bar{F}_{\text {tot }}$ are plotted against $\bar{\delta}$ in Figure 3 for the four models, based on which several conclusions can be drawn. Firstly, for a larger value of $\bar{\delta}$, the $\bar{F}_{\text {tot }}$ given by the three $\bar{F}_{\text {tot }}^{H}, \bar{F}_{\text {tot }}^{D M T}$ and $\bar{F}_{\text {tot }}^{J K R}$ are similar from each other, and $\bar{F}_{\text {tot }}^{B D}$ is much smaller than three $\bar{F}_{\text {tot }}^{H}, \bar{F}_{\text {tot }}^{D M T}$ and $\bar{F}_{\text {tot }}^{J K R}$. Secondly, the repulsive force of BD model and DMT model, exactly following the Hertzian equation. Thirdly, the magnitude of the attractive force of BD model is much higher than that given by the JKR and DMT theories. For instance, $\bar{F}_{a}^{B D}$ at $\bar{\delta}=3$ is about two times of $\bar{F}_{a}^{J K R}$ but increases to about three times at $\bar{\delta}=3$. The deviation between $\mathrm{BD}$ and JKR, in terms of the difference $\bar{F}_{a}^{B D}-\bar{F}_{\text {tot }}^{J K R}$, becomes even more significant as the overlap further increases.

Before proceeding, it is stated that the current model could be easily applied to the particle-surface normal contact. For a particle with radius $r_{1}$ impacting normally with a surface with radius $r_{2}$, the effective radius $R^{*}$ certainly reduces to $r_{1}$, i.e., $r_{2}=+\infty$. Similarly effective mass $\mathrm{m}^{*}$ reduces to $m_{1}$. 
Figure 3. Comparison of the variation of attractive force and overall force with the overlap in the models of Hertz, DMT, BD and JKR, under the static contact circumstance.

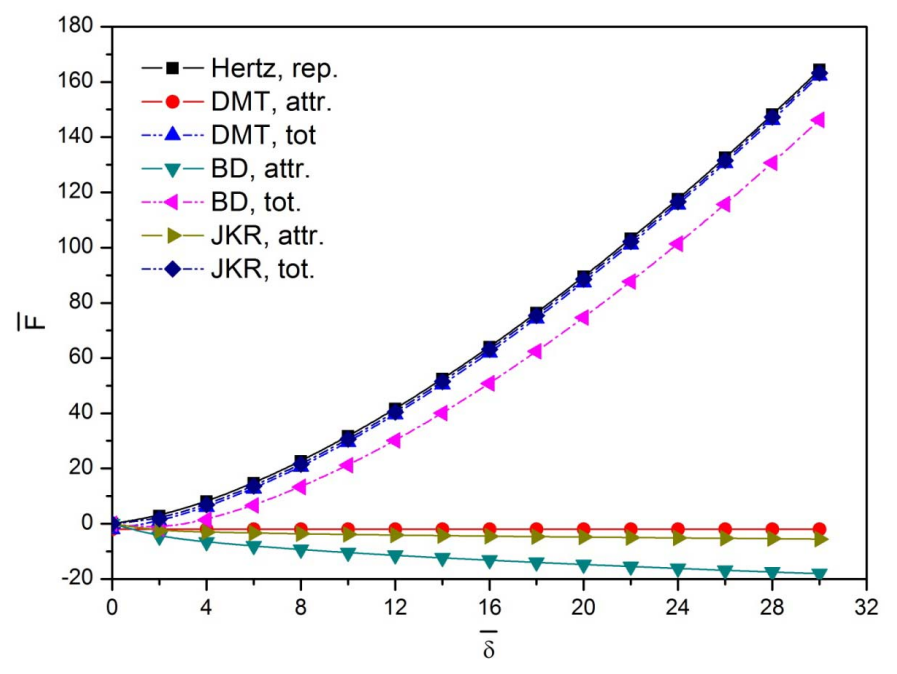

\subsection{Energy Dissipation}

Before proceeding, the contact theories between interacting particles are built on the static or quasi-static condition. However, the particle impacting process is the dynamic problem. The energy dissipation theory is introduced in order to study the parameter variation of particle impacting process.

A complete impact process includes the approaching phase and the rebound phase. During the approaching phase, the contact area between interacting particles gradually increased, and kinetic energy of the incident particle is converted to the strain energy and the acoustic wave energy, etc. [7]. During the rebound phase, the strain energy stored is partly converted to kinetic energy of the rebound particle, while another portion is dissipated. Certain amount of energy is lost by the fraction of $1-v_{r}{ }^{2} / v_{i}{ }^{2}$ with respect to the initial kinetic energy $1 / 2 m v_{i}^{2}$, where $m$ is particle mass, $v_{i}$ and $v_{r}$ are the particle velocities before and after the impact. From a macroscopic view, the energy loss is due to the force-deformation hysteresis of the impact procedure. It is known that the viscoelastic effect and the plastic deformation play import roles in energy loss during low-velocity impact and high-velocity impact, respectively $[7,10]$. If the relative impact velocity is just large enough to initiate yield in one of the spheres, the plastic deformation occurs at the center of contact area. The plastic deformation region gradually increases with increasing relative impact velocity, and until closes to the edge of the deformation region. When the present paper predicts the critical velocity, the plastic effect is considered to be less important and hence neglected.

Figure 4 shows the force displacement relationship as defined by Thornton and Zemin [18]. According to JKR theory, the contact of two colliding bodies commences when the overlap exceeds zero (point A in Figure 4), due to Van der Waals attractive force. When the contact force reaches a maximum value (point B in Figure 4) the particle velocity has been reduced to zero and the incoming stage is complete. In the recovery stage, all the work done during the loading stage has been recovered when point $\mathrm{A}$ is reached during the recovery stage. However, at this point, when $\delta=0$, the particle remains adhered to the surface and further work is required to separate the surfaces. Figure 4 shows that separation occurs at point $\mathrm{C}$ and hence the work required breaking the contact, which results in an energy loss given by: 


$$
W_{s}=7.09\left(\gamma^{5} R^{4} E^{-2}\right)^{1 / 3}
$$

where $\gamma, R$ and $E$ are the surface adhesive energy, reduced particle radius and Young's modulus, respectively [18]. When the energy losses are neglected by elastic wave propagation, the only work dissipated during a collision is the work done of separating the surfaces Ws. While the particle is captured, the critical impact velocity $v_{s}$ is obtained as:

$$
v_{s}=\left(14.18 / m_{p}\right)^{1 / 2}\left(\gamma^{5} R^{4} E^{-2}\right)^{1 / 6}
$$

below which the impact results in a final capture. For a particle impacting a flat surface, leading to:

$$
v_{s}=1.84(\gamma / R)^{5 / 6} /\left(\rho^{3} E^{2}\right)^{1 / 6}
$$

Figure 4. Schematic illustration of the force displacement relationship.

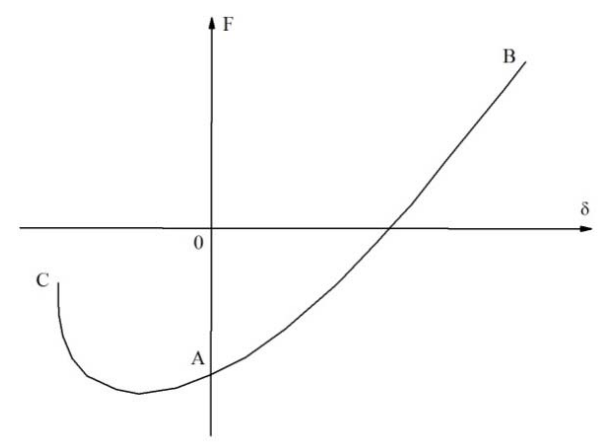

\subsection{Models Solution}

Using discrete method, the equation of particle motion is rewritten in a differential form as:

$$
\frac{\delta_{i}-2 \delta_{i+1}+\delta_{i+2}}{\Delta t^{2}}+\frac{\eta}{m_{1}} \frac{\delta_{i+1}-\delta_{i}}{\Delta t}+\frac{F_{t o t i+1}}{m_{1}}=0
$$

The differential equation is calculated from $\delta=0$ at $t=0$ to the maximum displacement of the impact and then toward the separation point $\delta=0$. The initial incident velocity of particle $v_{0}$ is obtained by the experiment. If the time step is small enough, the deformation of particle is small enough at time $t_{1}$. It can be found that motion of the particle considers to uniform motion at $\Delta t=t_{1}-t_{0}$. Therefore, the displacement at time $t_{1}$ is written as $\delta_{1}=v_{0} \Delta t$. Then, substituting $\delta_{1}$ and $F_{\text {tot, } 1}$ into Equation (31) we obtain $\delta_{2}$, and so on until the end of the impaction.

Firstly, assuming damping coefficient is a constant at time $t_{0}$. Then, the rebound velocity is calculated by Equation (31). Finally, $v_{n} / v_{0}$ is obtained by the calculation comparison with the normal restitution coefficient is obtained by the experiment. When the difference between the two data of restitution coefficient is large, re-adjusting the damping factor, until the two data of restitution coefficient are equal or very close to the end of the calculation.

\section{Results and Discussion}

In this section, the impact process of fly ash particles is first investigated through experimental methods. Secondly, variation of damping coefficient $(\eta)$ with normal incident velocity $\left(v_{i n}\right)$ is developed 
based on experimental results and impact dynamics models. Thirdly, model prediction on critical velocity is computed and with compare with experiments. Finally, the variation of contact displacement with impact contact time is obtained.

\subsection{Experimental Results}

The normal coefficient of restitution $e_{n}$ is defined as a ratio of the normal rebound $v_{r n}$ and the normal incident velocity $v_{i n}$. Figure 5 shows $e_{n} v s . v_{i n}$ for fly ash particles of diameter $88 \mu \mathrm{m}, 96 \mu \mathrm{m}$, $104 \mu \mathrm{m}$. For all cases, the impact with normal incident velocity lower than the critical velocity leads to a final capture $\left(e_{n}=0\right)$. For $v_{i n}$ larger than but close to $v_{c}$, the $e_{n} \sim v_{i, n}$ curves has a steep increase. However, its slope rapidly decreases as $v_{i n}$ further increases, and the $e_{n}$ rapidly decreases with increasing $v_{i n}$. The normal restitution coefficient of fly ash particle shows some difference than that of elastic spheres, especially at higher incident velocities.

Further analysis from a macroscopic view, the energy loss is due to the force-deformation hysteresis of the impact procedure. The viscoelastic effect is considered to be more important with the incident velocity close to and not significantly higher than the critical velocity. When the incident velocity gradually increases, the plastic deformation occurs in the fly ahs particle around the local contact area, and viscoelastic effect is still considered to be more important. The viscoelastic dissipation $\left(w_{s}\right)$ can be written $W_{s}=7.09\left(\gamma^{5} R^{4} E^{-2}\right)^{1 / 3}$, where $\gamma, \mathrm{R}$ and $\mathrm{E}$ are the surface adhesive energy, particle radius and Young's modulus, respectively [18]. It can be found that the viscoelastic dissipation is independent with incident velocity. However, the rebound kinetic energy of particle increases with increasing incident velocity, and leading to normal restitution coefficient increases. On the other hand, when the incident velocity exceeds the yield limit velocity, the contact area due to permanent plastic deformation gradually increases with incident velocity. Therefore, normal restitution coefficient decreases with increasing incident velocity.

Figure 5. The normal coefficient of restitution, $e_{n}$, versus the incident normal velocity $v_{i n}$ for fly ash particles of diameter $88 \mu \mathrm{m}, 96 \mu \mathrm{m}, 104 \mu \mathrm{m}$.

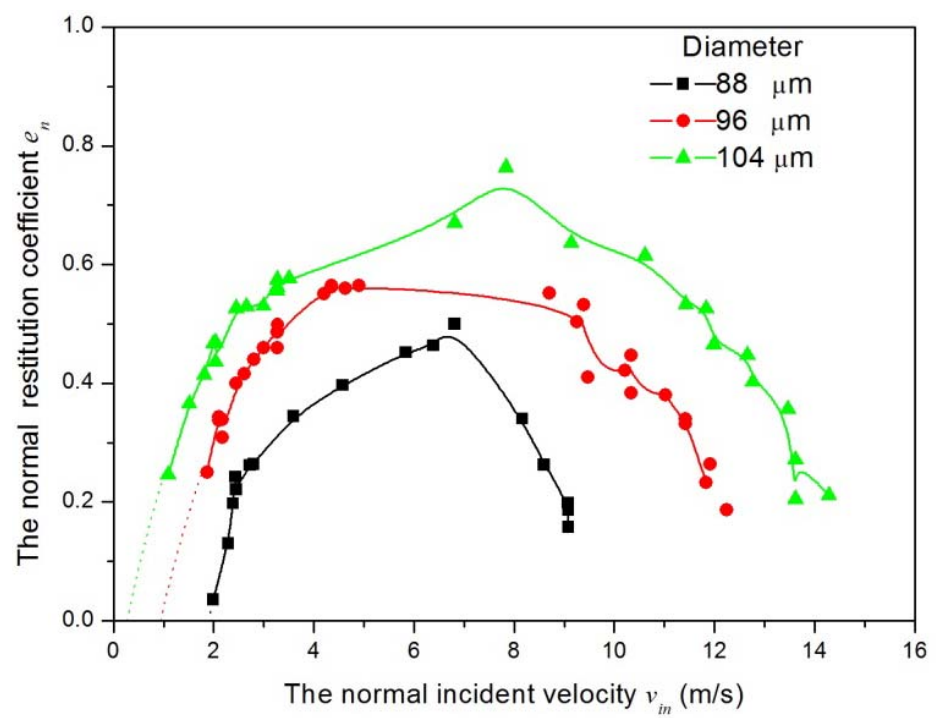




\subsection{Dependence of Damping Coefficient on Incident Velocity}

Besides the physical properties such as adhesion energy, Young's modulus, particle size and density, the damping coefficient is the most important parameter affecting the dynamic model predictions. However, it remains a challenge to directly determine the damping coefficient according to the physical properties of the impacting bodies in present state. Commonly, the damping coefficients are found by matching the predicted $e \sim v_{i}$ variation with experimental results [11,33]. However, in their model damping coefficients are kept constant for same particle diameter. As an idealization, the damping force is assumed to be linearly dependent on both its origin force and the relevant velocity [34]. That is the damping coefficient changes with the incident velocity of particle. Therefore, it is necessary to calculate the damping coefficients of different velocity conditions. In this section, the damping coefficient is calculated from Equation (31) based on the experimental results.

Before proceeding, the Hertz model only considers the repulsive force in the contact area of particle with target surface. However, the DMT and BD models consider repulsive force and attractive force. The repulsive forces of the BD model and DMT model exactly follow the Hertzian equation. For the DMT model, attractive force is idealized by an annular force at the perimeter of the contact area. For the BD model, attractive force is idealized by a line force at the perimeter of the contact area.

For the Hertz model, substituting $\mathrm{F}_{\text {tot }}{ }^{\mathrm{H}}$ into Equation (31), the equation of particle motion is given as:

$$
\frac{\delta_{i}-2 \delta_{i+1}+\delta_{i+2}}{\Delta t^{2}}+\frac{\eta}{m_{1}} \frac{\delta_{i+1}-\delta_{i}}{\Delta t}+\frac{4}{3} \frac{E^{*} r_{1}^{1 / 2}}{m_{1}} \delta_{i+1}^{3 / 2}=0
$$

For the DMT model, substituting $\mathrm{F}_{\text {tot }}{ }^{\mathrm{DMT}}$ into Equation (31), the equation of particle motion is given as:

$$
\frac{\delta_{i}-2 \delta_{i+1}+\delta_{i+2}}{\Delta t^{2}}+\frac{\eta}{m_{1}} \frac{\delta_{i+1}-\delta_{i}}{\Delta t}+\frac{4}{3} \frac{E^{*} r_{1}^{1 / 2}}{m_{1}} \delta_{i+1}^{3 / 2}-\frac{2 \pi w r_{1}}{m_{1}}=0
$$

For the $\mathrm{BD}$ model, substituting $\mathrm{F}_{\text {tot }}^{\mathrm{BD}}$ into Equation (31), the equation of particle motion is given as:

$$
\frac{\delta_{i}-2 \delta_{i+1}+\delta_{i+2}}{\Delta t^{2}}+\frac{\eta}{m_{1}} \frac{\delta_{i+1}-\delta_{i}}{\Delta t}+\frac{4}{3} \frac{E^{*} r_{1}^{1 / 2}}{m_{1}} \delta_{i+1}^{3 / 2}-\frac{2 \pi f r_{1}^{1 / 2}}{m_{1}} \delta_{i+1}{ }^{1 / 2}=0
$$

The proposed dynamic model is now used to calculate variation of damping coefficient with incident velocity. For three fly ash particle diameters $d_{p}(88 \mu \mathrm{m}, 96 \mu \mathrm{m}$ and $104 \mu \mathrm{m})$ and three models (Hertz model, DMT model and BD model), the evolution curves of damping coefficient $\eta$ with incident velocity $v_{\text {in }}$ are shown in Figure 6 . It is seen that the variation of $\eta$ with $v_{i n}$ can be roughly divided into the three parts.

(i) In the first part, $\eta$ decreases with increasing $v_{i n}$. However, $e_{n}$ increases with increasing $v_{i n}$. For three particle diameters, the variation tendency of damping coefficient with normal incident velocity is similar in those three models. When the normal incident velocity is small, the damping coefficient magnitude of the BD model is much smaller than both the Hertz model and DMT model for the same normal incident velocity. When the normal incident velocity is gradually increased, the damping coefficient calculated based on the three models tend to consistent for the same normal incident velocity.

In order to deeply understand the relationship between the damping coefficient and the normal incident velocity, in the following, we calculate the contact time under different normal incident velocity conditions, and examine the influence of the normal incident velocity on the damping coefficients. It can 
be seen from Figure 7 that in the first part, the contact time has a slightly downward tendency with the increasing normal incident velocity. While the normal incident velocity is close to about $2 \mathrm{~m} / \mathrm{s}$, the contact time is $294 \mathrm{~ns}, 232 \mathrm{~ns}$ and $224 \mathrm{~ns}$ for particle diameters of $88 \mu \mathrm{m}, 96 \mu \mathrm{m}$ and $104 \mu \mathrm{m}$, respectively. As discussed above, the viscoelastic effect is considered to be more important with the incident velocity close to and not significantly higher than the critical velocity. Therefore, the viscoelastic effect increases with decreasing normal incident velocity, and contact time is longer, and the damping coefficient is greater.

Figure 6. The damping coefficient variation with normal incident velocity for (a) Particle diameter: $88 \mu \mathrm{m}$; (b) Particle diameter: $96 \mu \mathrm{m}$; (c) Particle diameter: 104 $\mu \mathrm{m}$.
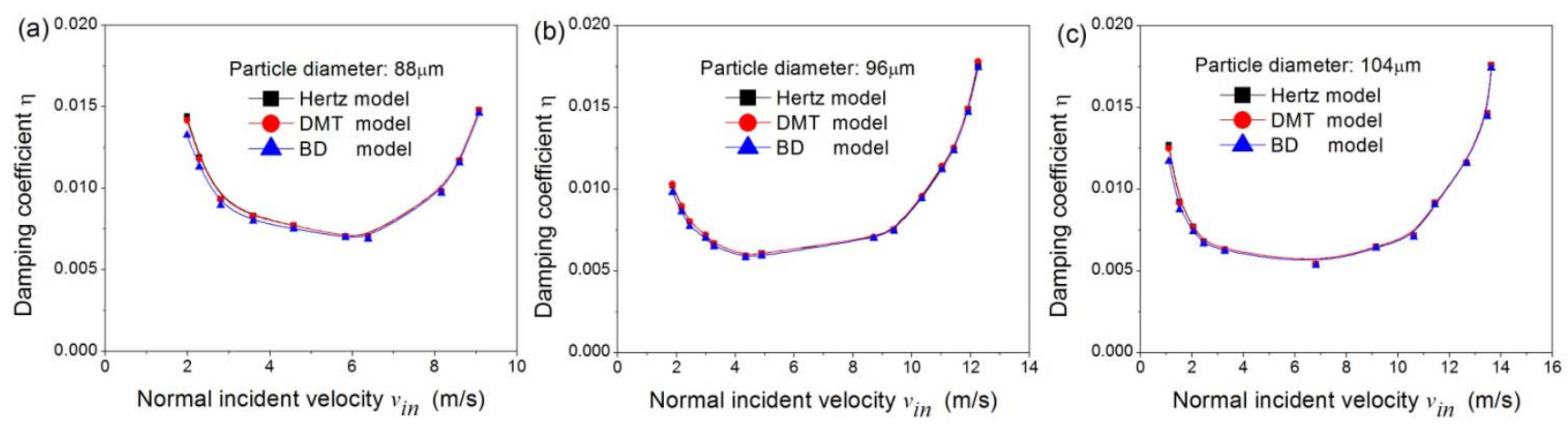

(ii) In the second part, $\eta$ is little changed with increasing $v_{i n}$, and $e_{n}$ is still little changed with increasing $v_{i n}$. For the same diameter of fly ash particle, the variation tendency of damping coefficient with normal restitution coefficient is similar in those three models. Damping coefficient calculated based on the three models is very close for the same normal restitution coefficient. Figure 7 shows that contact time is little changed with increasing $v_{i n}$ in second part. In this part, the contact area due to permanent plastic deformation gradually increases with increasing incident velocity. The effect of plastic deformation on the energy dissipation increases with increasing normal incident velocity. Therefore, the normal restitution coefficient, the damping coefficient and the contact time are all little changed with increasing $v_{\text {in }}$ in second part. The second part is a transitional range.

Figure 7. The contact time variation with incident normal velocity for (a) Particle diameter: $88 \mu \mathrm{m}$; (b) Particle diameter: $96 \mu \mathrm{m}$; (c) Particle diameter: $104 \mu \mathrm{m}$.
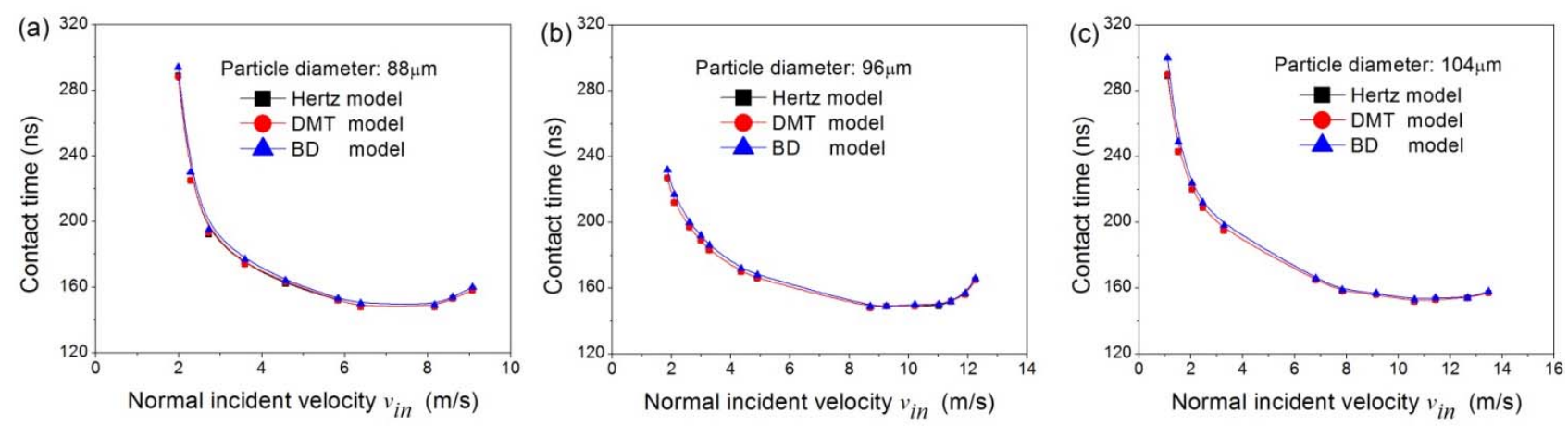

(iii) In the third part, $\eta$ rapidly increases with increasing $v_{i n}$. However, $e_{n}$ decreases with increasing $v_{i n}$. For the same diameter of fly ash particle, the variation tendency of damping coefficient with normal 
restitution coefficient is similar as those three models. Comparing Figure 6 with Figure 7, we can find a contact time rising tendency with the increasing normal incident velocity, implying that the plastic deformation plays important roles in energy loss and the energy loss due to viscoelastic effect could be negligible when the incident velocity is continually increased.

In order to understand effect of the particle diameter on the damping coefficient, the evolution curves of damping coefficient variation with incident normal velocity of different size particles for BD model are shown in Figure 8. Figure 8 shows that the damping coefficient increases with the decreasing particle size, and it is consistent with of $e_{n}-v_{i n}$ variation at same impact velocities.

Figure 8. The damping coefficient variation with incident normal velocity of different size particles for BD model.

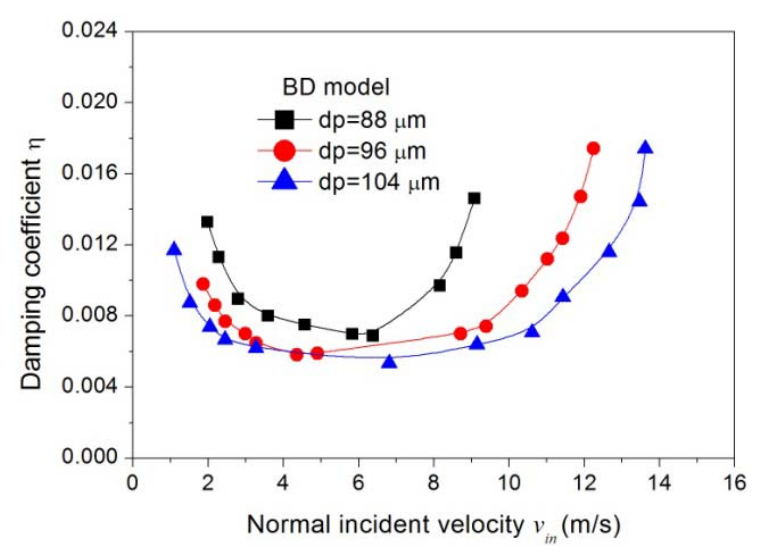

\subsection{Critical Normal Impact Velocity}

If the incident velocity is below the critical impact velocity, the particle will be captured by the substrate surface. The magnitude of critical impact velocity reflects the relative strength of adhesion. For the incident velocity close to the critical velocity, the plastic effect is considered to be neglected.

According to the results of Figures 5 and 6, using generalized least squares we can fit the relationships between damping coefficient and normal restitution coefficient, which are within the range of normal restitution coefficient increases with increasing incident velocity in Figure 5. The fitting relationships are shown in Table 2. When the normal restitution coefficient is 0 , the critical damping coefficient can be obtained by the fitting relationships in Table 2. The critical damping coefficients of different model are shown in Table 3.

Table 2. The fitting relationships of damping coeefficient and normal restitution coefficient.

\begin{tabular}{cccc}
\hline $\begin{array}{c}\text { Models } \\
\text { dp }(\boldsymbol{\mu m})\end{array}$ & Hertz model & DMT model & BD model \\
\hline 88 & $\eta=0.0238 \mathrm{e}_{\mathrm{n}}{ }^{2}-0.0291 \mathrm{e}_{\mathrm{n}}+0.0153$ & $\eta=0.0223 \mathrm{e}_{\mathrm{n}}{ }^{2}-0.0279 \mathrm{e}_{\mathrm{n}}+0.0151$ & $\eta=0.0193 \mathrm{e}_{\mathrm{n}}{ }^{2}-0.0247 \mathrm{e}_{\mathrm{n}}+0.0141$ \\
96 & $\eta=0.0104 \mathrm{e}_{\mathrm{n}}{ }^{2}-0.0229 \mathrm{e}_{\mathrm{n}}+0.0155$ & $\eta=0.0115 \mathrm{e}_{\mathrm{n}}{ }^{2}-0.0235 \mathrm{e}_{\mathrm{n}}+0.0156$ & $\eta=0.0101 \mathrm{e}_{\mathrm{n}}{ }^{2}-0.0213 \mathrm{e}_{\mathrm{n}}+0.0149$ \\
104 & $\eta=0.0074 \mathrm{e}_{\mathrm{n}}{ }^{2}-0.0217 \mathrm{e}_{\mathrm{n}}+0.0163$ & $\eta=0.0065 \mathrm{e}_{\mathrm{n}}{ }^{2}-0.0206 \mathrm{e}_{\mathrm{n}}+0.016$ & $\eta=0.0043 \mathrm{e}_{\mathrm{n}}{ }^{2}-0.017 \mathrm{e}_{\mathrm{n}}+0.0145$ \\
\hline
\end{tabular}


Table 3. The critical damping coefficients of different model.

\begin{tabular}{cccc}
\hline Particle diameter $(\boldsymbol{\mu m})$ & Hertz model & DMT model & BD model \\
\hline 88 & 0.0153 & 0.0151 & 0.0141 \\
96 & 0.0155 & 0.0156 & 0.0149 \\
104 & 0.0163 & 0.0160 & 0.0145 \\
\hline
\end{tabular}

Substituting $e_{n}=0$ and critical damping coefficients into Equations (32)-(34) we obtain initial incident velocity, which is predicted by Equations (32)-(34) as the critical velocity. The critical velocities of different model are shown in Table 4.

Table 4. Critical capture velocity of different model.

\begin{tabular}{ccccc}
\hline Particle diameter $(\boldsymbol{\mu m})$ & Hertz model $(\mathbf{m} / \mathbf{s})$ & DMT model $(\mathbf{m} / \mathbf{s})$ & BD model $(\mathbf{m} / \mathbf{s})$ & Experimental results \\
\hline 88 & 1.57 & 1.85 & 1.93 & 1.92 \\
96 & 0.71 & 1.05 & 1.27 & 1.25 \\
104 & 0.42 & 0.64 & 0.72 & 0.69 \\
\hline
\end{tabular}

Table 4 shows that the critical velocity decreases with increasing particle size. Critical velocity is the smallest predicted by the Hertz model for the same particle size. The reason is that Hertz model only considers the repulsive forces between particles and surfaces. However, the DMT model and BD model consider repulsive forces and attractive forces of the total force between particles and surfaces. In addition, the DMT model considers the attractive force to be lower than the BD model, therefore the critical velocity calculated by the BD model is higher.

For the same material particle, Equation (30) can be simplified to $v_{s} \infty R^{-5 / 6}$. Liu et al. [13] shows that $W S$ is introduced into the total energy loss once the contact is established, and refer $W_{S}$ as the first-contact energy loss for simplicity. The critical velocity determined solely by the first-contact energy loss, is proportional to $d_{p}{ }^{-5 / 6}$ and therefore gets larger for smaller particles. For instance, in the present work, the velocity $v_{c}$ of the particle with diameter of $104 \mu \mathrm{m}$ is $0.72 \mathrm{~m} / \mathrm{s}$, which increases to $1.93 \mathrm{~m} / \mathrm{s}$ for the particle with diameter of $88 \mu \mathrm{m}$ for the BD model.

Comparing the critical velocity values obtained by the three models with that obtained by experiment; we can find the results calculated by the BD model are close to experiment, which shows the BD model is applicable for describing the interaction behavior between ash particles and planar surfaces. Based on further analysis of Figure 3, the magnitude of the attractive force of the BD model is much higher than that given by the JKR and DMT theories. Generally, the line force approximation adopted by the BD model has overestimated the adhesion force. According to this, the BD model is applicable for describing the interaction behavior between ash particles and planar surfaces in the present conditions.

\subsection{Contact Displacement-Contact Time Curve}

For fly ash particle diameters of $88 \mu \mathrm{m}, 96 \mu \mathrm{m}$ and $104 \mu \mathrm{m}$, the contact displacement-contact time curves of the fly ash particle impacting a planar surface are plotted in Figure 9, where we take the critical incident velocity, incident velocity in the curves of $e_{n}$ increases with increasing $v_{i n}$, incident velocity in the curves of $e_{n}$ decreases with increasing $v_{i n}$. Here, the displacement is defined by $\delta=\mathrm{r}-\mathrm{d}$, where $r$ is the particle radius, and $d$ is the distance from particle center to planar surface. In addition, 
numerical calculation is based on $\mathrm{BD}$ model. The displacement curves with increasing and decreasing contact time correspond to the incoming and rebounding stages, respectively.

It can be seen from Figure 9, the contact time in the incoming stage is larger than that in the rebounding stage. In order to understand the deference of contact time in the two stages, we introduce the momentum analysis of the impact process. The Equation (2) shows that the damping force of impact process is:

$$
\begin{gathered}
\vec{F}^{D}=\vec{\eta} \frac{d \delta}{d t} \\
\int_{0}^{t_{m}}\left(\vec{F}_{t o t}+\vec{F}^{D}\right) d t=-m \vec{v}_{i n}
\end{gathered}
$$

where $t_{m}$ is contact time of incoming stage. The momentum equation of rebounding stage is given by:

$$
\int_{t_{m}}^{t_{n}}\left(\vec{F}_{t o t}+\vec{F}^{D}\right) d t=m \vec{v}_{r n}
$$

where $t_{n}$ is cut-off time of impact process.

Considering the direction of the force and velocity, substituting Equation (35) into Equations (36) and (37) we obtain:

$$
\begin{gathered}
\int_{0}^{t_{m}} \vec{F}_{t o t} d t=\vec{F}_{t o t, i} t_{m}=m v_{i}-\eta \delta_{\max } \\
\int_{t_{m}}^{t_{n}} \vec{F}_{t o t} d t=\vec{F}_{t o t, r}\left(t_{n}-t_{m}\right)=m v_{r}+\eta \delta_{\max }
\end{gathered}
$$

where $\vec{F}_{t o t, i}$ and $\vec{F}_{t o t, r}$ are the average of total force of incoming and rebounding stages; $\delta_{\max }$ is the maximum contact displacement. $\vec{F}_{t o t, i}$ and $\vec{F}_{t o t, r}$ can be considered equal approximately we obtain:

$$
\vec{F}_{t o t, i}=\vec{F}_{t o t, r}=F_{A}
$$

Comparing Equations (38) and (39) we obtain:

$$
F_{A}\left[\left(t_{n}-t_{m}\right)-t_{m}\right]=2 \eta \delta_{\max }-\left(m v_{i n}-m v_{r n}\right)
$$

In order to obtain value of the right side of Equation (41), we introduce the following inequality:

$$
\begin{aligned}
& \eta \delta_{\max }\left(v_{i n}+v_{r n}\right)=\eta v_{i n} \int_{0}^{t_{m}} v d t+\eta v_{r n} \int_{0}^{t_{n}} v d t=\eta \int_{0}^{t_{m}} v_{i n} v d t+\eta \int_{0}^{t_{n}} v_{r n} v d t \\
& >\eta \int_{0}^{t_{m}} v v d t+\eta \int_{0}^{t_{n}} v v d t=\eta \int_{0}^{t_{m}} v v d t+\eta \int_{0}^{t_{n}} v v d t=\eta \int_{i} \frac{d \delta}{d t} d \delta+\eta \int_{r} \frac{d \delta}{d t} d \delta
\end{aligned}
$$

with the subscripts $i$ and $r$ representing the incoming and rebounding stages, respectively. However, the work done of damping force in the incoming and rebounding stages equals the difference between incident kinetic energy and rebound kinetic energy, we consequently obtain:

$$
\eta \int_{i} \frac{d \delta}{d t} d \delta+\eta \int_{r} \frac{d \delta}{d t} d \delta=\frac{1}{2} m v_{i n}^{2}-\frac{1}{2} m v_{r n}^{2}
$$

Substituting Equation (43) into Equation (42) we obtain: 


$$
2 \eta x_{m}>\left(m v_{i}-m v_{r}\right)
$$

Substituting Equation (44) into Equation (41) we obtain:

$$
t_{n}-t_{m}>t_{m}
$$

Therefore, the contact time in the incoming stage is larger than that in the rebounding stage. In addition, it can be seen from Figure 9, the difference of contact time in the incoming and rebound stages decreases with increasing incident velocity.

For the critical incident velocity, it can be seen from Figure 9, that the contact time is $419 \mathrm{~ns}, 521 \mathrm{~ns}$ and $532 \mathrm{~ns}$ for particle diameter of $88 \mu \mathrm{m}, 96 \mu \mathrm{m}$ and $104 \mu \mathrm{m}$, respectively. The contact time increases with increasing particle diameter. In addition, the variation trend of contact displacement with contact time tends to have gentle slope at the cut-off time, the slope of the curve tends to be 0 , and that shows the fly ash particle is captured.

For the normal incident velocity is greater than the critical incident velocity, the maximum contact displacement increases with increasing incident velocity. In addition, the slope of the contact displacement curve is less than 0 at the cut-off time, and that shows the fly ash particle rebound from the planar surface. For the same normal incident velocity, the maximum contact displacement increases with increasing diameter.

Figure 9. The contact displacement variation with contact time of BD model for (a) Particle diameter: $88 \mu \mathrm{m}$; (b) Particle diameter: $96 \mu \mathrm{m}$; (c) Particle diameter: $104 \mu \mathrm{m}$.
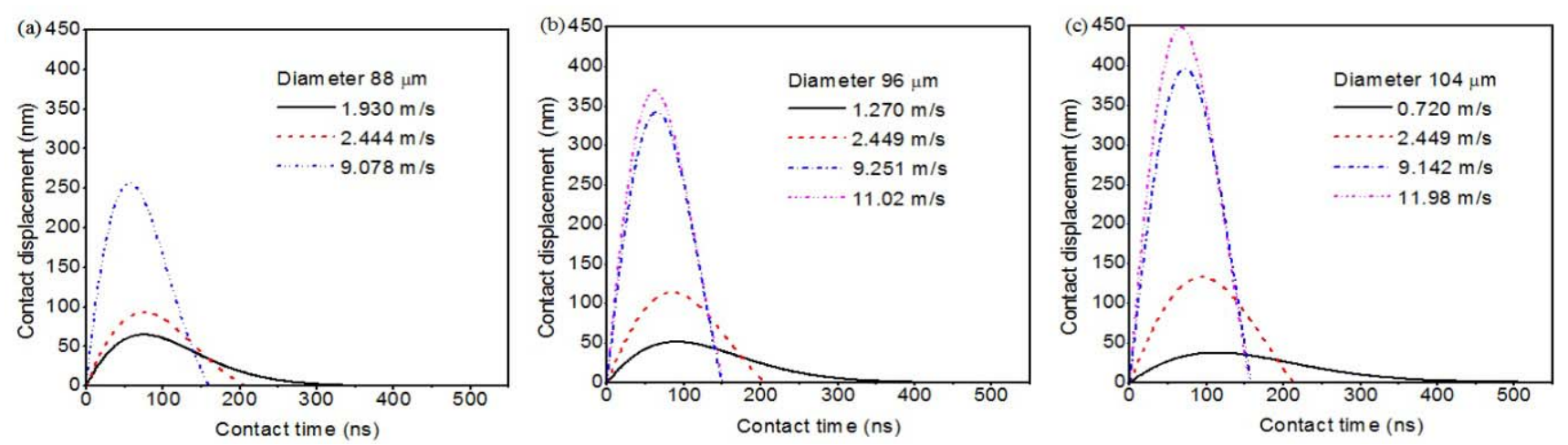

\section{Conclusions}

The normal impact behavior of fly ash particles on a planar surface has been investigated using the dynamic model with the energy dissipation. The following conclusions can be drawn from our calculations:

(1) The impact of fly ash particles with a rigid substrate shows some differences than that of elastic spheres, especially at higher incident velocities. For fly ash particles, it can be found that the normal restitution coefficient rapidly increases with increasing incident velocity for the incident velocity is less than the yield velocity, and rapidly decreases with increasing incident velocity for the incident velocity is greater than the yield velocity.

(2) The variation of damping coefficient with normal incident velocity can be roughly divided into three parts. In the first part, damping coefficient decreases with increasing normal incident velocity. In the second part, damping coefficient is little changed with increasing normal incident 
velocity. In the third part, damping coefficient rapidly increases with increasing normal incident velocity. In all parts, damping coefficient calculated based on the three models tend to be consistent for the same normal incident velocity.

(3) For lower incident velocities, viscoelastic dissipation plays a more important role in the impact process than plastic deformation. The contact time increases with decreasing incident velocity. On the other hand, when the incident velocity exceeds the yield limit velocity, plastic deformation dissipation dominates the impact behavior. The contact time increases with increasing the normal incident velocity.

(4) The critical velocity decreases with increasing particle size. Comparing the critical velocities obtained by the three models with that obtained by experiment; we can find the results calculated by the BD model are close to the experimental value, which shows the BD model is applicable for describing the interaction behavior between ash particles and planar surfaces. Generally, the line force approximation adopted by the BD model has overestimated the adhesion force. According to this, the BD model is applicable for describing the interaction behavior between ash particles and planar surfaces under the present conditions.

(5) The contact time in the incoming stage is larger than that in the rebounding stage, and the difference of contact time in the incoming and rebound stages decreases with increasing incident velocity. For the critical incident velocity, the contact time increases with increasing particle diameter. For the normal incident velocity is greater than the critical incident velocity, the maximum contact displacement increases with increasing incident velocity. For the same normal incident velocity, the maximum contact displacement increases with increasing diameter.

\section{Acknowledgments}

This work is supported by National Natural Science Foundation of China (NSFC Grant No. 51006055) and by the Fundamental Research Funds for the Central Universities (No. DUT11RC (3) 27).

\section{Conflict of Interest}

The authors declare no conflict of interest.

\section{References:}

1. Losurdo, M.; Spliethoff, H.; Kiel, J. Ash deposition modeling using a visco-elastic approach. Fuel 2012, 102, 145-155.

2. Baxter, L.L.; De Sollar, R.W. A mechanistic description of ash deposition during pulverized coal combustion: Predictions compared with observations. Fuel 1993, 72, 1411-1418.

3. Kostoglou, M.; Konstandopoulos, A.G. Particulate deposit shape evolution on cylinders in cross-flow at high stokes numbers. J. Aerosol Sci. 1999, 31, 427-436.

4. Brach, R.M.; Dunn, P.F. A mathematical model of the impact and adhesion of microspheres. Aerosol Sci. Technol. 1992, 16, 51-64.

5. Dahneke, B. Measurements of the bouncing of small latex spheres. J. Colloid Interface Sci. 1973, 45, 584-590. 
6. Dahneke, B. Further measurements of the bouncing of small latex spheres. J. Colloid Interface Sci. 1975, 51, 58-65.

7. Rogers, L.N.; Reed, J. The adhesion of particles undergoing an elastic-plastic impact with a surface. J. Phys. D Appl. Phys. 1984, 17, 677-689.

8. Dunn, P.F.; Brach, R.M.; Caylor, M.J. Experiments on the Low-Velocity Impact of Microspheres with Planar Surfaces. Aerosol Sci. Technol. 1995, 23, 80-95.

9. Kim, O.V.; Dunn, P.F. Direct visualization and model validation of microsphere impact and surface capture. J. Aerosol Sci. 2008, 39, 373-375.

10. Wall, S.; John, W.; Wang, H.C.; Goren, S.L. Measurements of kinetic energy loss for particles impacting surfaces. Aerosol Sci. Technol. 1990, 12, 926-946.

11. Brach, R.M.; Dunn, P.F. Macrodynamics of microparticles. Aerosol Sci. Technol. 1995, 23, 51-71.

12. Konstandopoulos, A.G. Deposit growth dynamics: Particle sticking and scattering phenomena. Powder Technol. 2000, 109, 262-277.

13. Guanqing, L.; Shuiqing, L.; Qiang, Y. A JKR-based dynamic model for the impact of micro-particle with a flat surface. Powder Technol. 2011, 207, 215-223.

14. John, W. Particle-surface interactions: charge transfer, energy loss, resuspension, and deagglomeration. Aerosol Sci. Technol. 1995, 23, 2-24.

15. Dahneke, B. Particle bounce or capture-search for an adequate theory: I. Conservation-of-energy model for a simple collision process. Aerosol Sci. Technol. 1995, 23, 25-39.

16. Andres, R.P. Inelastic energy transfer in particle/surface collisions. Aerosol Sci. Technol. 1995, $23,40-50$.

17. Kostoglou, M.; Konstandopoulos, A.G. Particulate deposit shape evolution oncylinders in cross-flow at high Stokes numbers. J. Aerosol Sci. 2000, 31, 427-436.

18. Thornton, C.; Ning, Z. A theoretical model for the stick/bounce behaviour of adhesive, elastic-plastic spheres. Powder Technol. 1998, 99, 154-162.

19. Cundall, P.A.; Strack, O.D.L. A discrete numerical model for granu-lar assemblies. Geotechnique 1979, 29, 47-65.

20. Marshall, J.S. Discrete-element modeling of particulate aerosol flows. J. Comput. Phys. 2009, $228,1541-1561$.

21. Shuiqing, L.; Marshall, J.S. Discrete element simulation of micro-particle deposition on a cylindrical fiber in an array. J. Aerosol Sci. 2007, 38, 1031-1046.

22. Cheng, W.; Brach, R.M.; Dunn, P.F. Three-dimensional modeling of microsphere contact/impact with smooth, flat surfaces. Aerosol Sci. Technol. 2002, 36, 1045-1060.

23. Kim, O.V.; Dunn, P.F. A microsphere-surface impact model for implementation in computational fluid dynamics. J. Aerosol Sci. 2007, 38, 532-549.

24. Johnson, K.L.; Kendall, K.; Roberts, A.D. Surface energy and the contact of elastic solids. Proc. R. Soc. Lond. A 1971, 324, 301-313.

25. Adb-Elhady, M.S.; Rindt, C.C.M.; Wijers, J.G.; van Steenhoven, A.A. Modelling the impaction of a micron particle with a powdery layer. Powder Technol. 2006, 165, 53-64.

26. Matsunaga, T.; Kim, J.K.; Rohatgi, P.K. Crystallinity and selected properties of fly ash particles. Mater. Sci. Eng. A 2002, 325, 333-343. 
27. Chung, F.H. Quantitative interpretation of X-ray diffraction patterns of mixtures. 11. Adiabatic principle of X-ray diffraction analysis of mixtures. J. Appl. Crystallogr. 1974, 7, 526-531.

28. Shackelford, J.F.; Alexander, W. The CRC Materials Science and Engineering Handbook; CRC Press: Boca Raton, FL, USA, 1992; pp. 436-438.

29. Johnson, K.L. Contact Mechanics; Cambridge University Press: Cambridge, UK, 1985.

30. Maugis, D. Contact, Adhesion and Rupture of Elastic Solids; Springer: New York, NY, USA, 2000.

31. Derjaguin, B.V.; Muller, V.M.; Toporov, Y.P. Effect of contact deformations on the adhesion of particles. J. Colloid Interface Sci. 1975, 53, 314-326.

32. Muller, V.M.; Yushchenko, V.S.; Derjaguin, B.V. On the influence of molecular forces on the deformation of elastic spheres and its sticking to a rigid plane. J. Colloid Interface Sci. 1980, 77, 91-101.

33. Li, X.; Dunn, P.F.; Brach, R.M. Experimental and numerical studies on the normal impact of microspheres with surfaces. J. Aerosol Sci. 1999, 30, 439-449.

34. Hunt, K.H.; Crossley, F.R.E. Coefficient of restitution interpreted as damping in vibroimpact. ASME J. Appl. Mech. 1975, 97, 440-445.

(C) 2013 by the authors; licensee MDPI, Basel, Switzerland. This article is an open access article distributed under the terms and conditions of the Creative Commons Attribution license (http://creativecommons.org/licenses/by/3.0/). 